\title{
Novel endothelial cell markers in hepatocellular carcinoma
}

\author{
Xin Chen ${ }^{1, *}$, John Higgins ${ }^{2, *}$, Siu-Tim Cheung ${ }^{3}$, Rui $\mathrm{Li}^{4,5}$, Veronica Mason ${ }^{2,5}$, \\ Kelli Montgomery ${ }^{2}$, Sheung-Tat Fan ${ }^{3}$, Matt van de Rijn ${ }^{2}$ and Samuel So ${ }^{4,5}$ \\ ${ }^{1}$ Department of Biopharmaceutical Sciences, University of California San Francisco, San Francisco, CA, \\ USA; ${ }^{2}$ Department of Pathology, Stanford University, Stanford, CA, USA; ${ }^{3}$ Department of Surgery, The \\ University of Hong Kong, Hong Kong, China; ${ }^{4}$ Department of Surgery and ${ }^{5}$ Asian Liver Center, Stanford \\ University, Stanford, CA, USA
}

\begin{abstract}
Hepatocellular carcinoma is characterized by hypervascularity and a propensity for vascular invasion. Detailed analysis of complementary DNA (cDNA) microarray global gene expression data and further validation on a smaller independent sample set by reverse transcription-polymerase chain reaction established the presence of two endothelial gene clusters in hepatocellular carcinoma. Cluster I, consists of 20 cDNA clones, representing 15 unique genes. Cluster II consists of nine unique genes. The expression of the cluster I genes appeared to be significantly upregulated in hepatocellular carcinoma compared with normal liver, cirrhotic liver, or nontumor liver tissues adjacent to the hepatocellular carcinoma. The pattern of gene expression of cluster I genes correlated positively with the 'proliferation gene cluster' and 'stromal cells cluster 2'. Expression of cluster II genes, in contrast, was not significantly different between hepatocellular carcinoma and non-neoplastic liver tissues. Studies conducted to localize the protein products of these genes by immunohistochemical staining of tissue arrays with up to 350 cores of tissues, and by in situ hybridization led to the discovery of novel sinusoidal endothelial cell markers in hepatocellular carcinoma: podocalyxin-like and regulator of G protein signaling-5. Our results underscore fundamental differences not only between neoplastic vs non-neoplastic liver cells but also between the hepatic sinusoidal endothelium of hepatocellular carcinoma and normal liver.

Modern Pathology (2004) 17, 1198-1210, advance online publication, 21 May 2004; doi:10.1038/modpathol.3800167
\end{abstract}

Keywords: microarray; HCC; endothelial cell; angiogenesis

Hepatocellular carcinoma (HCC) is the most common adult liver malignancy and ranks among the top five causes of cancer death in the world. ${ }^{1}$ Chronic infection with hepatitis B virus (HBV) and hepatitis $\mathrm{C}$ virus (HCV) are the most important risk factors of HCC globally and in the United States. ${ }^{2}$ However, the molecular mechanism of this association remains largely unknown. ${ }^{3}$ Patients with cirrhosis as a result of HBV or HCV infection are at higher risk of HCC compared with those with less advanced chronic liver disease. ${ }^{4}$ In the United States, the incidence of HCC is 2.4 per 100000 and is rising. ${ }^{5}$ Treatment options for liver cancer are currently very limited. Surgical resection or liver transplantation for early stage tumors is considered

Correspondence: Professor S So, MD, FACS, Department of Surgery, Asian Liver Center and Stanford University School of Medicine, 300 Pasteur Drive, H3680 Stanford, CA 94305, USA. E-mail: samso@stanford.edu

*These authors contributed equally to this work.

Received 16 December 2003; revised 9 February 2004; accepted 16 February 2004; published online 21 May 2004 the only curative treatment in HCC. ${ }^{6}$ However, because HCC tumors are generally asymptomatic during the early stages, more than $80 \%$ of patients have unresectable tumors at the time of diagnosis. ${ }^{7}$ Systemic chemotherapy and/or radiotherapy are not effective treatment for HCC. Hence, HCC remains one of the deadliest cancers with a 5-year survival rate of only $5 \% .^{8}$

Endothelial cells line the normal hepatic sinusoid. These cells contain small fenestrations to allow the free diffusion of macromolecules, but not of large particles such as chylomicrons, between the blood and the hepatocyte surface. Physiologically, these cells play important roles in filtration and endocytotic functions. They are also active in trafficking secretory products of the hepatocytes. ${ }^{9,10}$ However, little is known about the molecular basis for the formation and function of sinusoidal endothelial cells.

Angiogenesis is required for a variety of physiological processes as well as the growth and progression of tumor cells. ${ }^{11,12}$ During the past several years, multiple factors, including vascular endothelial cell 
growth factor (VEGF) and angiopoietin, have been associated with angiogenesis in HCC. ${ }^{13,14}$ Studies in mouse tumor models showed that there could be many differences in the structure of tumor vs normal vessels. For example, tumor vessel walls could be abnormally permeable as a result of endothelial fenestrae, vesicles and transcellular holes, widened interendothelial junctions, and discontinuous basement membrane. ${ }^{15,16}$ All these defects may make tumor vessels leaky, which may in turn facilitate tumor metastasis. ${ }^{17}$ While there are fundamental differences in endothelial cells in tumors and those in normal human tissues, little is known about the molecular biology of the endothelial cells in HCC. Only a few well-characterized endothelial cell markers, including CD34, CD31 and von Willebrand factor (vWF), have been studied in HCC. CD34 is known to be strongly expressed in the sinusoidal endothelial cells in HCC by immunohistochemistry (IHC) compared with nontumor liver tissues, whereas antibodies against CD31 and vWF only stain a small number of sinusoidal endothelial cells in HCC and at lower intensity. ${ }^{18,19}$

Recent evidence suggests that the phenotypic diversity of tumors is associated with corresponding diversity in their gene expression programs. cDNA microarray technology has been used to study gene expression patterns in different tumor types and has provided new insight into the development and classification of these cancers. These studies also help to elucidate novel clinical biomarkers for early detection and prognostication. ${ }^{20-23}$ In our previous study, we described the systematic characterization of the global gene expression patterns in human liver cancers using cDNA microarrays containing 23000 clones, representing approximately 17600 genes. ${ }^{24,25}$ We showed that each HCC has its own distinct gene expression pattern, and HCC and nontumor tissues can be readily distinguished based on their global gene expression profiles. We were able to identify a large number of genes that were differentially expressed in HCC and nontumor tissues, including a 'proliferation cluster' that was upregulated in HCC and a 'liver-specific cluster' that was downregulated relative to non-neoplastic liver. Also, there appeared to be two endothelial gene clusters that have not been studied. In this paper, we report the detailed analysis and characterization of these endothelial cell markers in HCC and nontumor liver tissues.

\section{Experimental methods}

\section{Liver Tissues and RNA Isolation}

The liver tissues were obtained from surgical resections or transplants performed at Stanford University, CA, USA or Queen Mary Hospital, The University of Hong Kong, Hong Kong, China. All patients gave informed consent before surgery. This study is approved by the Institutional Review Board at Stanford University and the ethics committee of The University of Hong Kong. All tissues were snap frozen in liquid nitrogen within $0.5 \mathrm{~h}$ after the surgery, and stored at $-80^{\circ} \mathrm{C}$. Frozen liver samples were used for cDNA microarray study and reverse transcription-polymerase chain reaction (RT-PCR); and paraffin-embedded samples were used for IHC and in situ hybridization (ISH). Total RNA was extracted from tissues using Trizol Reagent (Invitrogen, Carlsbad, CA, USA). For microarray analysis, messenger RNA (mRNA) was isolated from total RNA using FastTract mRNA purification kit (Invitrogen).

\section{cDNA Microarray Procedure and Data Analysis}

The method of complementary DNA (cDNA) microarray analysis of the liver tissues has already been described. ${ }^{24}$ In this study, the array data were retrieved for further analysis from the Stanford Microarray Database (http://genome-www.stanford. edu/microarray). ${ }^{26}$ All nonflagged array elements for which the fluorescent intensity in each channel was greater than 1.5 times the local background were considered well measured. Genes for which fewer than $75 \%$ of measurements across all the samples in this study met this standard were excluded from further analysis. In this study, 3964 cDNA clones, representing about 3180 genes whose expression level differed by at least three-fold in at least four samples from their mean expression level across all samples were analyzed. We applied a hierarchical clustering algorithm both to the genes and arrays using the Pearson correlation coefficient as the measure of similarity, and average linkage clustering, as described. ${ }^{27}$ The results were visualized and analyzed with the publicly available software program TreeView (M Eisen; http://rana.lbl.gov).

To calculate the average correlation between gene clusters, the expression levels of the $20 \mathrm{cDNA}$ clones in endothelial cell cluster I and the nine clones in cluster II were averaged to obtain the average expression vector. The Pearson's correlation coefficient was then calculated for every clone in the whole cluster (comprised of 3964 clones) against the individual average expression vector. To obtain the correlation curve, the moving average of 21 clones was calculated and plotted against the main cluster (along the vertical axis) as described. ${ }^{28}$

\section{RT-PCR}

RT-PCR was performed using ThermoScripte RTPCR system (Invitrogen). In brief, $1 \mu \mathrm{g}$ of total RNA was used in a $20 \mu \mathrm{l}$ reverse transcription assay. In all, $2 \mu \mathrm{l}$ of the reverse transcription product was subsequently used in the $25 \mu \mathrm{l}$ PCR reaction. All PCR 
Table 1 Primer sequences of RT-PCR

\begin{tabular}{lll}
\hline Gene name & Forward primer & Reserve primer \\
\hline Actin & TCGTGCGTGACATTAAGGAG & ATGCCAGGGTACATGGTGGT \\
CD31 & TAGCGCATGGCCTGGTTAGAG & GGCGGTGCTCCCAAGTAGTCT \\
CD34 & GCCTCCCAAGTTTAGGAC & GGCAACAGCTCAACCCA \\
CDH5 & CCTGCCATGCCAGTAACTGT & ATATCTTGGGTTCCTCTAAC \\
Col15A1 & TTGTTGTTGAGTGTAAGATGTCCT & AATTGTGGGGTGGGGGTTGG \\
EDG1 & ACTGAGCCAAAGGTCTAGCAT & TCGGCTTCAATGGATATG \\
FKBP12 & TCAGCTTTGCTTCCGACA & CCCAACACCAATTCCTATTCT \\
INTA7 & TTGGGATGAAGAGGGTAGAGT & GACTGATGGCAGCAAACTAAG \\
MSTP032 & GGCAAACGAGAGCAATGACTA & GTTGGACCCAAATATGTTAAA \\
PODXL & AATTCCTTTCCCAGTTGT & TTCTCAGTAAATTCCAGTGTA \\
RGS5 & TCCACCTGCCCATAGTCA & TGCAGGAAGCGAAATAGTGAA \\
SPARCL1 & TGGCACTGACAATCAGACCTA & TCAAAGAAACGGGTTAT \\
vWF & CATGACACTGAAGCGTGATGA & GCCTGGCAGTGATGTCGTT \\
\hline
\end{tabular}

was performed at $52-55^{\circ} \mathrm{C}$ annealing temperature for 25-32 cycles. The primer sequences for PCR for each of the genes are available in Table 1.

\section{Tissue Microarray Construction and IHC}

IHC was performed as previously described using microwave heat-induced epitope retrieval in citrate buffer. $^{29}$ In order to avoid interference from endogenous biotin, a biotin-free method, EnVision ${ }^{\mathrm{TM}}$, was used for amplification of the signal (Dako, Carpinteria, CA, USA). The following available antibodies were used: CD34 (Dako, 1:1000), vWF (Dako, 1:400), and podocalyxin-like (PODXL) (AGI, Sunnyvale, CA, USA; 1:600).

Tissue microarrays were constructed as previously described. ${ }^{30,31}$ We used two tissue microarrays for this study. The first of these consisted of archival tissue retrieved from the surgical pathology files. This array contained 350 tissue cores each measuring $0.6 \mathrm{~mm}$ from 145 unique tissues. They included: seven normal livers, eight cirrhotic livers, 48 hepatocellular carcinomas, and 82 control tissues that included kidney, placenta, etc. The second tissue microarray contained 30 larger $2 \mathrm{~mm}$ cores that represented 28 unique tissues. These included: foir normal livers, one cirrhotic liver, 11 hepatocellular carcinomas, and other control tissues.

Tissue arrays were scored using a four-tier scale: 0-negative, 1-insufficient or equivocal, 2-weak staining, and 3-strong staining as previously reported. ${ }^{32}$ Only strong, extensive staining of sinusoidal endothelium was scored as strong positive. For the final data analysis, the average score for duplicate or triplicate tissue cores was calculated. Only the staining results of liver tissues (hepatocellular carcinoma, cirrhosis, and normal liver) were analyzed. Results of IHC on tissue microarrays were evaluated using cluster analysis with the assistance of the TMA-Deconvoluter. ${ }^{33}$ This software permits modification of tissue microarray data for hierarchical cluster analysis using the program Cluster and display using the program TreeView.

\section{Nonradioactive ISH of Paraffin Sections}

Nonradioactive ISH was performed as described. ${ }^{34}$ Digoxigenin (DIG)-labeled sense and antisense RNA probes were generated by PCR amplification of 400$600 \mathrm{bp}$ products with the T7 promoter incorporated into the primers. In vitro transcription was performed with DIG RNA labeling kit and T7 polymerase according to the manufacturer's protocol (Roche Diagnostics, Indianapolis, IN, USA). Sections (5 $\mu \mathrm{m}$ thick) were cut from the paraffin blocks, deparaffinized in xylene, and hydrated in graded concentrations of ethanol for $5 \mathrm{~min}$ each. Sections were incubated with $1 \%$ hydrogen peroxide, followed by digestion in $10 \mu \mathrm{g} / \mathrm{ml}$ of proteinase $\mathrm{K}$ at $37^{\circ} \mathrm{C}$ for $30 \mathrm{~min}$. Sections were hybridized overnight at $55^{\circ} \mathrm{C}$ with either sense or antisense riboprobes at $200 \mathrm{ng} / \mathrm{ml}$ dilution in mRNA hybridization buffer (Dako). The following day, sections were washed in $2 \times$ SSC and incubated with 1:35 dilution of RNase A cocktail (Ambion, Austin, TX, USA) in $2 \times$ SSC for $30 \mathrm{~min}$ at $37^{\circ} \mathrm{C}$. Next, sections were stringently washed in $2 \times \mathrm{SSC} / 50 \%$ formamide twice, followed by one wash at $0.08 \times \mathrm{SSC}$ at $50^{\circ} \mathrm{C}$. Biotin-blocking reagents (Dako) were applied to the section to block the endogenous biotin. For signal amplification, a horseradish peroxidase (HRP)-conjugated rabbit anti-DIG antibody (Dako) was used to catalyze the deposition of biotinyl-tyramide, followed by secondary streptavidin complex (GenPoint kit; Dako). The final signal was developed with DAB (GenPoint kit; Dako), and the tissues were counterstained in hematoxylin for $15 \mathrm{~s}$. The primer sequences used for the generation of probes are available in Table 2. The scoring and clustering of ISH of the liver tissue sections were the same as described in IHC although the signal achieved by ISH was often weaker than that seen by IHC. 
Table 2 Primer sequences for generating probes of ISH (not including T7 promoter sequence)

\begin{tabular}{lll}
\hline Gene name & Forward primer & Reverse primer \\
\hline RGS5 & TACTGTCCGAAGGCCTTCTT & GTCTAGCAGCATAAATACAT \\
MSTP032 & ATCACTTACACATCCCAAAA & CACTTCTACTTCATTCTCCT \\
CDH5 & AATTCATTCTGGAGGGGCAG & ACAATCCCTTGCAGTGTGAG
\end{tabular}

\section{Results}

\section{Identification of Distinct Endothelial Gene Clusters by cDNA Microarray Analysis}

As described in an earlier overview on the global gene expression profile of HCC, there appeared to be two distinct endothelial gene clusters in our analysis of HCC and non-HCC liver tissues ${ }^{20}$ (Figure 1a and b). The entire list of the $29 \mathrm{cDNA}$ clones that have not been previously published are shown in Table 3 . One of the endothelial gene clusters, designated cluster I, consists of $20 \mathrm{cDNA}$ clones, representing 15 unique genes. The second cluster, designated cluster II, consists of nine unique genes (Table 3). Apart from the well-known endothelial cell markers, CD34 and vWF, the expression of the other genes identified in both clusters have not been studied in HCC. They include genes whose expression in endothelial cells is either known or unknown. THY1 and SPARCL1 (also known as hevin), were identified as colon cancer endothelial cell markers using serial analysis of gene expression (SAGE).$^{35}$ COL15A1 was reported to stain capillary endothelium. ${ }^{36,37}$ Endothelial differentiation gene 1 (EDG1) was cloned from human umbilical vein endothelial cells and was implicated in differentiation of endothelial cells and vascular maturation. ${ }^{38,39}$ PODXL, a transmembrane protein with a similar structure to CD34, is expressed in the foot processes of glomerular podocytes and endothelial cells of normal kidney. ${ }^{40,41}$ Genes whose expression has not been described in endothelial cells include RGS5 and MSTP032.

With clustering analysis, cluster I genes are found to be highly expressed in HCC, whereas cluster II genes showed variable expression patterns in both neoplastic and non-neoplastic liver tissues (Figure 1b). Using the microarray data from 58 patients where paired HCC and adjacent nontumor liver samples were analyzed, the average gene expression level for each endothelial cell cluster was measured for both HCC and the paired nontumor liver tissues. For each endothelial cluster, the gene expression ratio for HCC vs nontumor liver tissue was calculated for each patient as represented in Figure 2a and b. Gene expression ratio for cluster I genes in paired HCC: nontumor liver was greater than 1.5 in $50 / 58$ or $86.2 \%$ (Figure 2a). The average ratio was 2.74, corresponding to an almost three-fold higher expression level of cluster I genes in HCC compared with the matched nontumor liver tissues from the same patient (Figure 2b). In contrast, the gene expression ratio for cluster II genes in HCC vs nontumor tissues was quite variable. Only $20 / 58$ or $34.5 \%$ showed an HCC/nonliver tissue ratio greater than 1.5 (Figure 2a), and the average ratio was 1.34 (Figure 2b).

In an attempt to identify features in the global gene expression cluster that may be associated with the expression profiles of the two endothelial cell clusters, we calculated correlation coefficients for the association of each gene expression pattern with the average expression vector of each cluster. The resulting correlation curves, plotted as moving averages (window size $=21$ genes), are displayed to the right in Figure 1a. We found that the genes in cluster II did not correlate with any other gene expression patterns in the global cluster. However, interesting correlations were observed with genes in cluster I. The genes in cluster I correlated positively with the 'proliferation cluster' and 'stromal cells cluster 2' (red arrows) and correlated negatively with 'liver-specific gene cluster' and 'stress response cluster' (green arrows).

\section{RT-PCR Analysis for the Expression Patterns of Endothelial Cell Markers in an Independent Sample Set}

To validate the differential expression pattern of endothelial genes in HCC found on microarray analysis, we studied an independent sample set of an additional 16 samples (frozen HCC and adjacent nontumor liver tissue from eight patients) by RTPCR. None of these samples were assayed in the cDNA microarray study. Five cluster I genes (CD34, Col15A1, MSTP032, PODXL, regulator of G protein signaling-5 (RGS5) and five cluster II genes (INTA7, hevin, EDG1, CDH5, vWF) were tested, and actin was used as an RNA loading control (Figure 3). The expression of CD31, a well-characterized endothelial cell marker, and FKBP12, a recently identified endothelial cell marker were also assayed. ${ }^{32}$

We found that the RT-PCR analysis in this independent sample set correlated with the differential endothelial genes expression pattern found by cDNA microarray analysis. Neither CD31 nor FKBP12 showed variable expression between HCC and nontumor liver tissues by RT-PCR, consistent with their expression patterns found in microarray analysis. As in the microarray analysis, there was a striking similarity between the expression levels of 
a

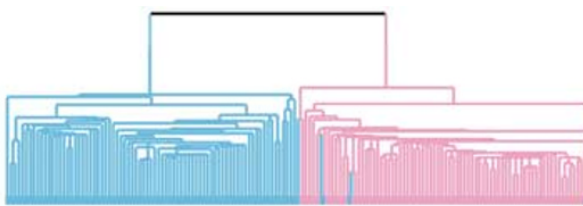

$\uparrow$

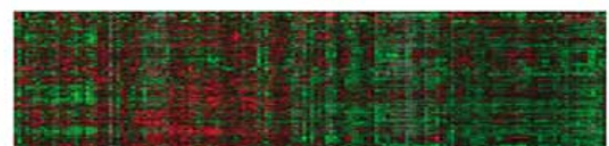

3964

CDNA

Clones

\rfloor

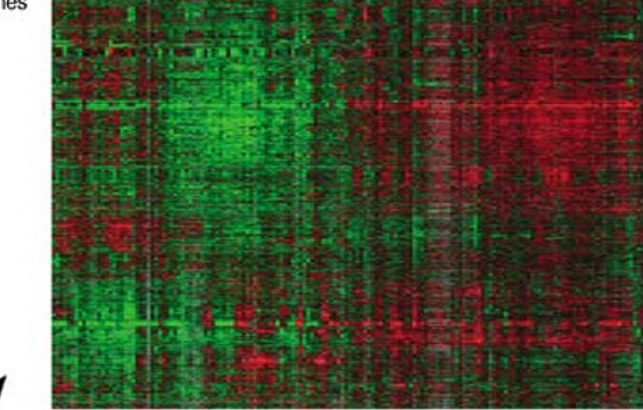

HCC

Non-tumor Liver

- Endothelial cluster I

I Proliferation

- Ribosomal proteins

Liver-specific

- Lymphocytes

- Stromal cells 1

Stress response

Stromal cells 2

Interferon response
Correlation coefficient
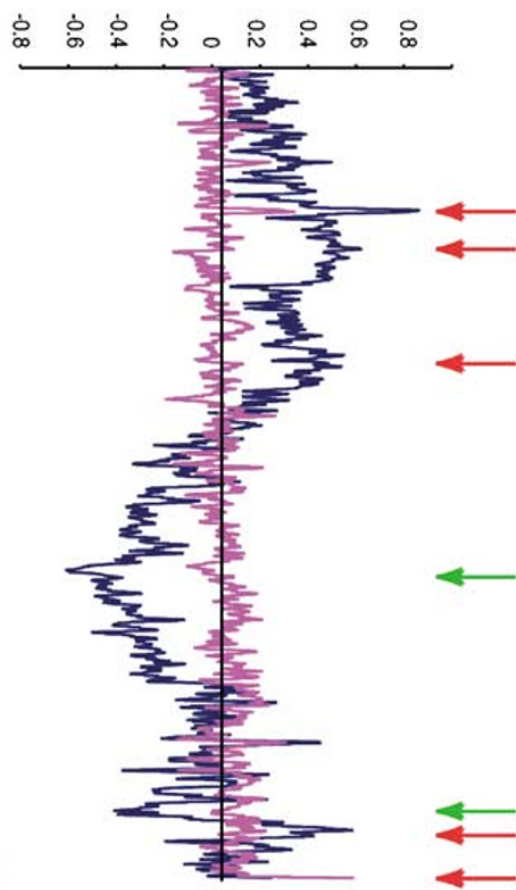

$\begin{array}{lllll}0.25 & 0.5 & 1 & 2 & 4\end{array}$

b

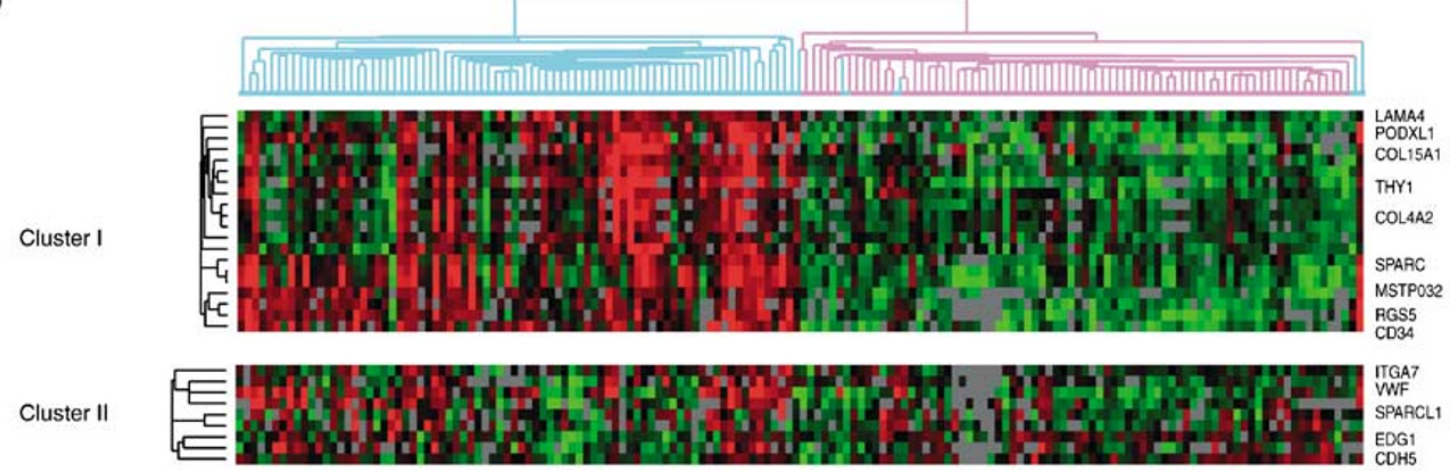

Figure 1 (a) Global gene expression patterns of liver tissues. Hierarchical clustering of the patterns of variation in expression of 3964 cDNA clones in 156 liver tissues $(74$ nontumor liver and 82 HCC) is shown to the left. The data are in a table format, in which rows represent individual genes and columns represent individual tissue sample. The color in each cell reflects the expression level of the corresponding gene in the corresponding tissue, relative to its mean expression level across the entire set of tissue samples. The scale (lower left corner) extends from fluorescence ratios of 0.25 to 4 relative to the mean level for all samples. Gray indicates missing or excluded data. Clusters corresponding to each individual feature of the expression patterns in liver tissues are labeled. The complete data set is available at the website: http://genome-www.stanford.edu/hcc/supplement.shtml, and http://genome-www5.stanford.edu/cgi-bin/ $\mathrm{SMD} /$ publication/viewPublication.pl?pub_no $=107$. The correlation values of each of the endothelial cell clusters are plotted as moving average of 21 genes (along the vertical axis) to the right. The blue line represents the moving average correlation to the average vector of genes in cluster I, whereas the pink line represents the moving average correlation to the average vector of genes in cluster II. The red arrows indicate positive correlations, and green indicates negative correlations. (b) Expanded view of endothelial cell cluster genes. The data are the same as (a), and only some of the gene names are shown due to the space limit. See Table 3 for the complete list of the genes. Part of Figure 1a and b published previously ${ }^{24}$ is reproduced with the permission by the American Society for Cell Biology.

the five cluster I genes. In six of the eight $(75 \%)$ HCC/nonliver tissue pairs, there was significantly higher expression of these genes in the HCC samples compared with the corresponding nontumor liver tissues. On the other hand, the expression levels of cluster II genes appeared to be much more variable, corresponding to the pattern seen in the microarray studies. Therefore, with RT-PCR, we were able to 
Table 3 List of clones in endothelial cell clusters

\begin{tabular}{|c|c|c|}
\hline Clone ID & Gene symbol/gene name & Accession number \\
\hline \multicolumn{3}{|c|}{ HCC endothelial cell cluster I } \\
\hline IMAGE:309826 & LAMA4 laminin, alpha 4 & N94616 \\
\hline IMAGE:290378 & PODXL podocalyxin-like & N64508 \\
\hline IMAGE:809901 & COL15A1 collagen, type XV, alpha 1 & AA455157 \\
\hline IMAGE:1323785 & THY1 Thy-1 cell surface antigen & AA 877226 \\
\hline IMAGE:81475 & EST & T63511 \\
\hline IMAGE:769686 & THY1 Thy-1 cell surface antigen & AA428836 \\
\hline IMAGE:1926620 & THY1 Thy-1 cell surface antigen & AI346653 \\
\hline IMAGE:769959 & COL4A2 collagen, type IV, alpha 2 & AA430540 \\
\hline IMAGE:293917 & SPARC, osteonectin & N66035 \\
\hline IMAGE:250654 & SPARC, osteonectin & H95959 \\
\hline IMAGE:487878 & SPARC, osteonectin & AA045463 \\
\hline IMAGE:882506 & LOXL2 lysyl oxidase-like 2 & AA676458 \\
\hline IMAGE:1584541 & EST & AA972348 \\
\hline IMAGE:341805 & MSTP032 & W60845 \\
\hline IMAGE:853809 & RGS5 regulator of G-protein signalling 5 & AA668470 \\
\hline IMAGE:271038 & RGS5 regulator of G-protein signalling 5 & N34362 \\
\hline IMAGE:898300 & EST & AA598822 \\
\hline IMAGE:666279 & EST & AA233805 \\
\hline IMAGE:666298 & EST & AA232840 \\
\hline IMAGE:770858 & CD34 CD34 antigen & AA434387 \\
\hline \multicolumn{3}{|c|}{ HCC endothelial cell cluster II } \\
\hline IMAGE:377671 & ITGA7 integrin, alpha 7 & AA055979 \\
\hline IMAGE:79190 & EST & AI821019 \\
\hline IMAGE:840486 & vWF von Willebrand factor & AA485883 \\
\hline IMAGE:823871 & SPARCL1 SPARC-like 1 (mast9, hevin) & AA490471 \\
\hline IMAGE:824659 & ICA1 islet cell autoantigen 1 & AA482169 \\
\hline IMAGE: 49710 & LAMP1 lysosomal-associated membrane protein 1 & $\mathrm{H} 28973$ \\
\hline IMAGE:26418 & EDG1 endothelial differentiation gene 1 & R13546 \\
\hline IMAGE:781468 & EST & AA431105 \\
\hline IMAGE:69672 & CDH5 cadherin 5, VE-cadherin & T53625 \\
\hline
\end{tabular}
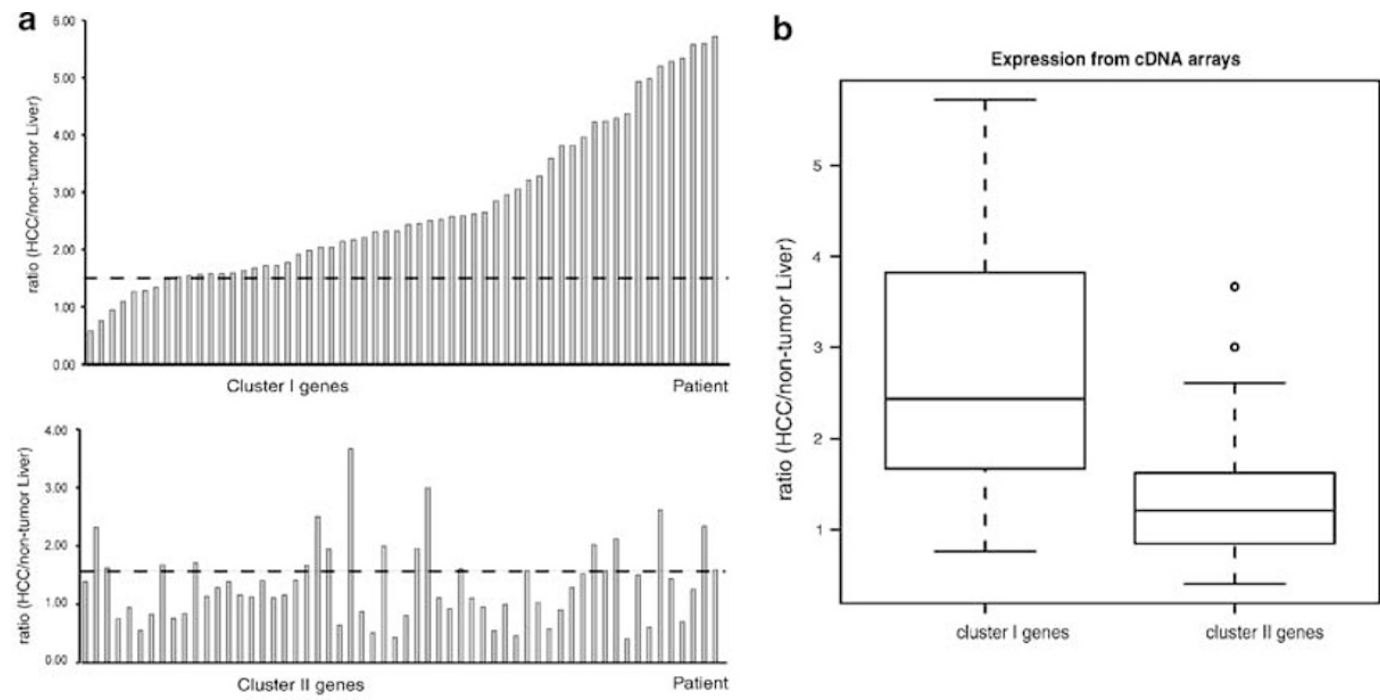

Figure 2 Relative gene expression patterns of endothelial cell cluster genes in nontumor and HCC patients. (a) Histogram of HCC/ nontumor liver ratio. Each bar represents the relative ratio of HCC/nontumor liver (from the same patient) of the average value of the cluster I genes (upper) and cluster II genes (lower). The patients are sorted according to the ratio of HCC/nontumor liver in cluster I genes, and displayed in the same order for cluters II genes. The dashed line represents that the average expression is 1.5-fold in HCC samples compared with the corresponding nontumor samples, which we used as an arbitrary cutoff for genes highly expressed in HCC. (b) The distribution of ratio of paired HCC/nontumor liver displayed as boxplots. The upper and lower boundaries of the box represent the interquartile range (IQR: the range between 25 th and 75 th percentiles), the line in the middle of the box represents the median. The upper and lower lines are $1.5 \times$ IQR. Values over or above 1.5IQR are plotted individually. 


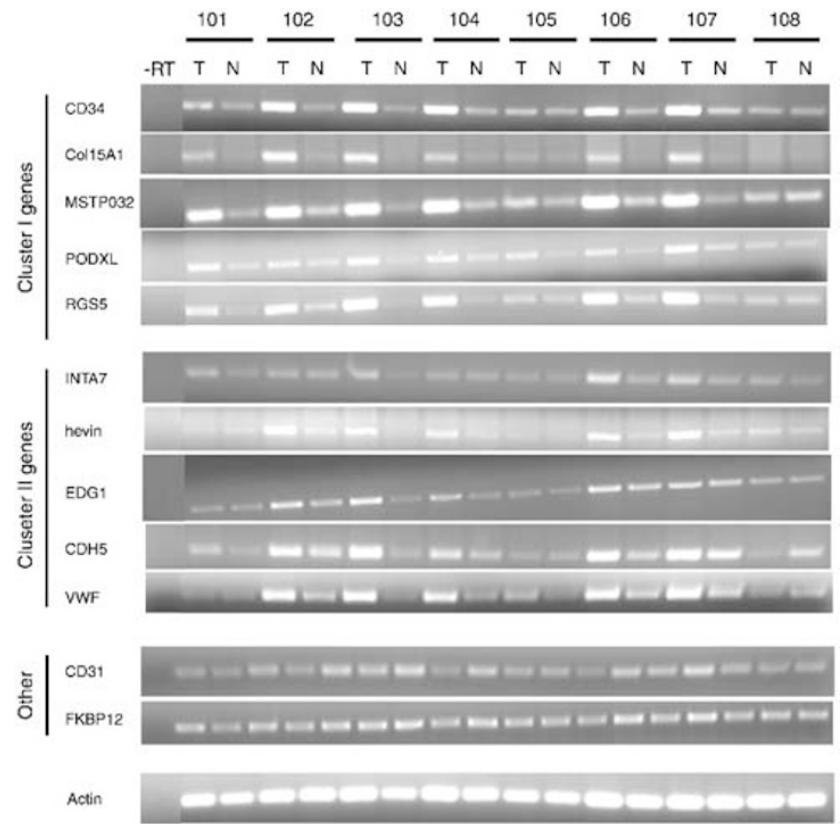

Figure 3 Confirmation of the endothelial cell markers identified from the microarray array experiments using RT-PCR. RT-PCR was used to analyze eight pairs of nontumor (N) and HCC (T) samples for five cluster I genes, five cluster II genes, and two other endothelial cell markers. None of those samples has been previously assayed by microarray. Actin is used as a loading control.

demonstrate using an independent sample set that the cluster I genes were significantly overexpressed in HCC samples. This result further demonstrates the robustness of the microarray technology to capture new gene expression signatures in tumor tissues.

\section{Immunohistochemical Staining of Endothelial Cell Markers on Tissue Sections}

We next investigated the expression patterns of these endothelial cell markers at the protein level in both conventional tissue sections and tissue microarrays. Three markers with available antibodies for immunohistochemical staining were studied: PODXL, CD34 (from cluster I) and vWF (from cluster II). PODXL has only been shown to be expressed by foot processes of glomerular podocytes and endothelial cells of normal kidney. ${ }^{40,41}$ The expression pattern of PODXL in liver tissues has not been reported.

Conventional tissue sections from 27 liver tissue samples obtained from 17 patients were used for immunohistochemical staining. They included paired HCC and adjacent cirrhotic liver samples from 10 patients, HCC from two patients, and normal liver samples from five patients. The results are shown in Table 4a and representative images are displayed in Figure 4. Among the 12 cases of HCC, PODXL showed diffuse, strong positive sinusoidal endothelial staining in eight, and focal staining in three cases. Only one case of HCC showed negative staining. PODXL was negative in all five normal
Table 4 IHC (a) and ISH (b) using conventional sections

(a) IHC

\begin{tabular}{llll}
\hline Endothelium from & CD34 & $v W F$ & PODXL \\
\hline Normal liver $(n=5)$ & 0 & 1str 1foc & 0 \\
Cirrhosis $(n=10)$ & 4foc & 4str 1foc & 2foc \\
Hepatocellular carcinoma & 10str 1 foc & 2str 3foc & 8str 3foc
\end{tabular}
$(n=12)$

(b) ISH

\begin{tabular}{llll}
\hline Endothelium from & MSTP032 & RGS5 & CDH5 \\
\hline Normal liver $(n=1)$ & 0 & 0 & 1foc \\
Cirrhosis $(n=3)$ & 0 & 0 & 2str \\
Hepatocellular carcinoma & 2str 1foc & 2str 1foc & 2str 1foc
\end{tabular}
$(n=3)$

str = diffuse strong staining of sinusoidal endothelial cells; $f o c=$ focal or weak staining of sinusoidal endothelial cells.

livers and 8/10 paired cirrhotic tissues. Only two cirrhotic livers showed focal sinusoidal staining for PODXL. CD34, the other endothelial gene in cluster I, showed a staining pattern that was similar to PODXL (Table 4a). In contrast to the staining pattern of PODXL and CD34, staining for VFW, a cluster II gene product, was highly variable in HCC and nonHCC liver tissues. VFW was strongly or focally positive in 2/5 normal livers, 4/10 cirrhotic livers, and 5/12 HCC.

\section{Immunohistochemical Staining of Endothelial Cell Markers on Tissue Microarrays}

Tissue microarrays that contained 350 tissue cores were then used to assay the expression patterns of these three endothelial cell markers. The staining patterns of 63 liver samples contained in the tissue arrays, including seven normal livers, nine cirrhotic livers, and 47 HCC were graded and a hierarchical clustering algorithm was applied for analysis (Figure 5a). Hierarchical analysis of the tissue array data directly corresponded to the pattern seen in gene expression or cDNA microarray analysis: CD34 and PODXL coclustered together in their staining patterns, while vWF was located in a separate branch.

Hierarchical analysis of the tissue microarrays also showed that the 63 liver tissues segregated into two branches (branches I and II, Figure 5a). Branch I consisted of liver tissues with strong positive staining for all three markers or for both CD34 and PODXL. Branch II contained liver tissues largely with negative staining for all three markers or strong staining for vWF. In total, $37 / 37$ or $100 \%$ of the liver tissues in branch I were HCC. In contrast, $16 / 26$ or $61.5 \%$ of the liver tissues in branch II were nontumor liver tissues. The distribution of HCC and nontumor liver tissues between branches I and II based on their pattern of staining for PODXL, 


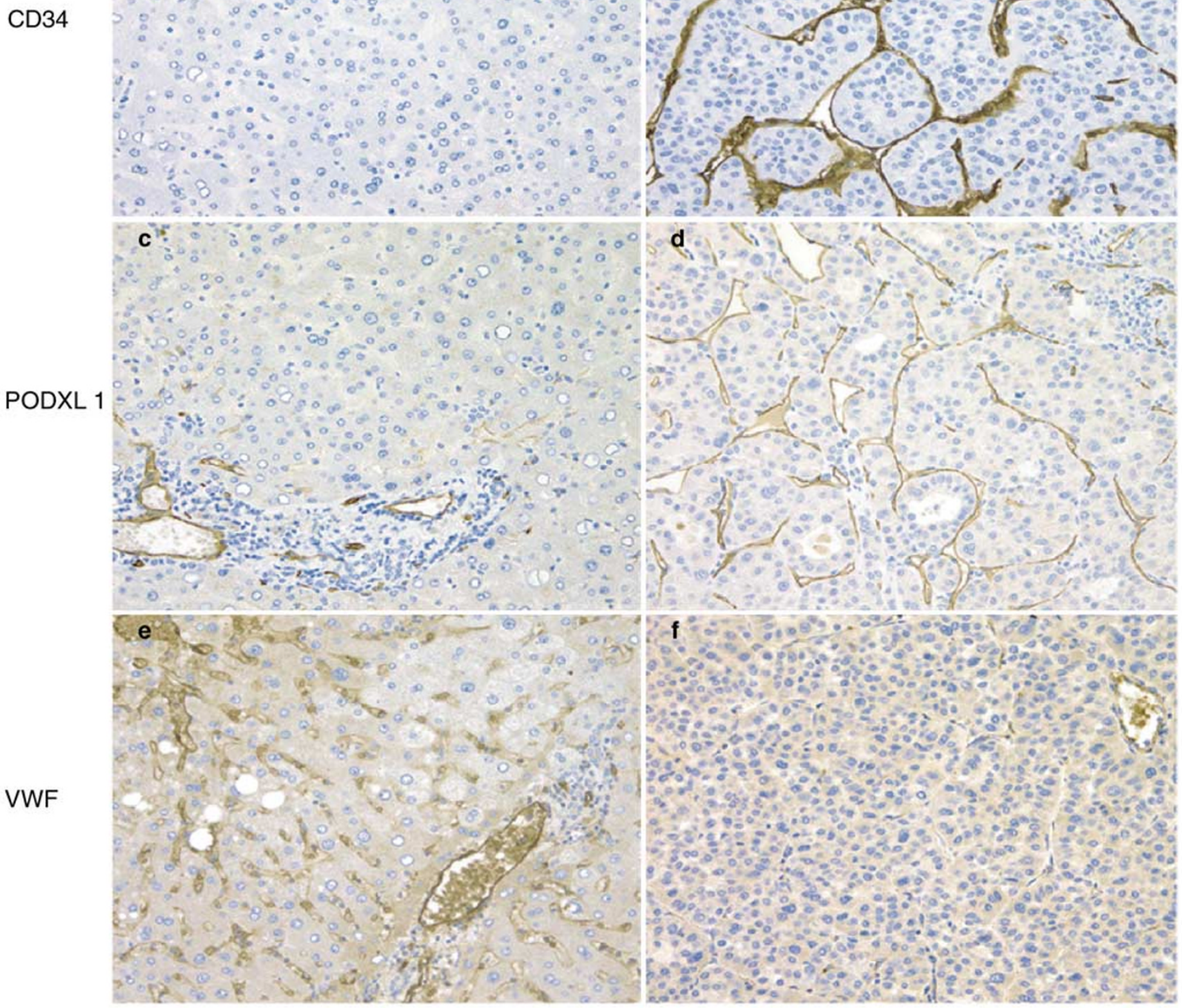

Figure 4 Immunohistochemical staining of endothelial cell markers. (a), (c), and (e) are normal liver sections; whereas (b), (d), and (f) are HCC sections. (a) and (b) were stained with anti-CD34 antibody (cluster I); (c) and (d) with anti-PODXL antibody (cluster I); (e) and (f) with anti-vWF antibody (cluster II). Both of the cluster I antibodies show strong staining of the endothelium between the trabeculae of malignant hepatocytes $(\mathbf{b}, \mathbf{d})$, but fail to stain the sinusoids in the normal liver (a, c). The antibody against vWF, in contrast, stains the endothelium in the normal liver but fails to highlight the endothelium in the HCC.

CD34, and VFW is highly statistically significant $(P<0.001$ by Fisher's exact test).

\section{ISH Study of Novel Endothelial Cell Markers}

MSTP032 and RGS5 were two cluster I genes that have not been reported to be associated with endothelial cells. RGS5 is a signal transduction molecule that has structural homology to SST2 of $S$. cerevisiae and EGL-10 of C. elegans. It has been shown to be expressed at high levels in heart, lung, skeletal muscle, and small intestine. ${ }^{42}$ Since there are no antibodies available for immunohistochemical staining, we used ISH to evaluate the expression of these two novel endothelial cell markers in HCC and nontumor liver tissues. In this study, we assessed the expression of MSTP032 and RGS5 (two cluster I genes) with CDH5, a cluster II gene. The results of ISH of the three genes in seven conventional liver sections (three HCC, three 


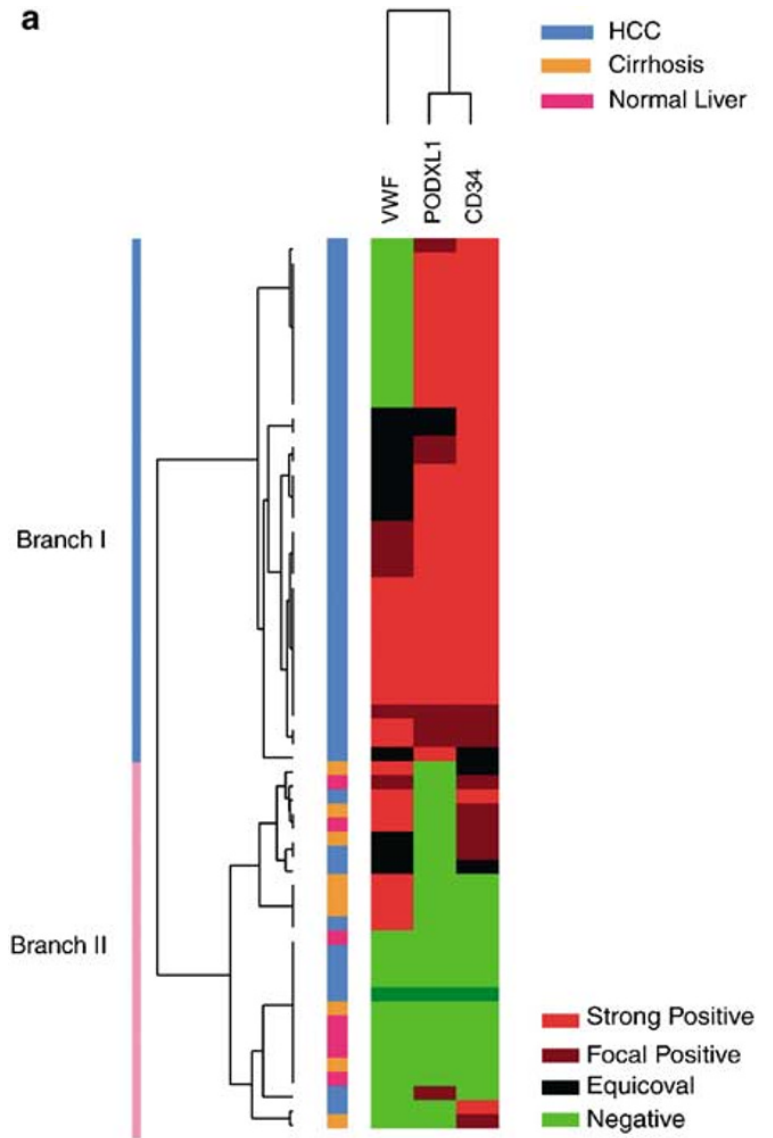

b

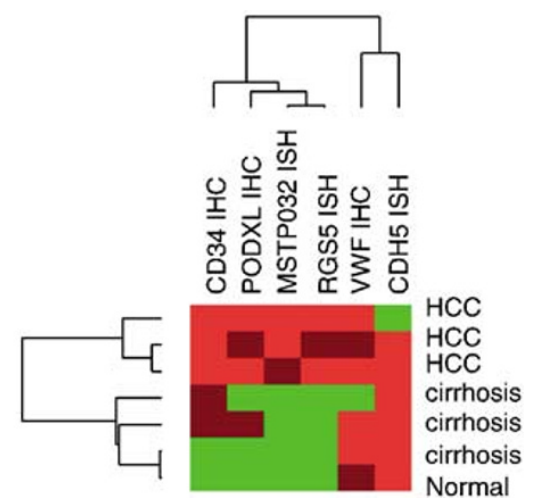

Figure 5 (a) Hierarchical clustering of the staining patterns of 63 liver samples using CD34, vWF, and PODXL. The data are shown in a table format, in which rows represent liver samples and columns represent antibodies. The color scale represents the relative staining patterns of each sample, and is depicted on the lower right side. The sample type (normal liver, cirrhosis, or HCC) is displayed between the cluster and dendrogram and annotated on the upper right corner. (b) Hierarchical clustering of the staining patterns of seven liver tissues using IHC and ISH.

cirrhotic, and one normal liver samples) are summarized in Table $4 \mathrm{~b}$.

ISH showed that RGS5 is not expressed in the sinusoidal endothelial cells in the non-tumor liver tissues; but its mRNA can be readily detected in the sinusoidal endothelial cells in HCC samples (Figure 6a and b). We found likewise, MSTP032 mRNA is not expressed in the normal sinusoidal endothelial cells, but is present in the sinusoidal endothelial cells in HCC samples (Figure 6c and d). The expression patterns of RGS5 and MSTP032 detected by ISH were similar to those observed by immunohistochemical staining of other cluster I genes, including PODXL and CD34. In comparison, CDH5 (present in cluster II) was detected in the sinusoidal cell of the normal liver, two of three cases of cirrhosis, and all three cases of HCC (Table $4 \mathrm{~b}$ and Figure 6e and f).

We next clustered the seven liver samples with immunohistochemical and in situ staining of all six markers (Figure 5b). Strikingly, the hierarchical clustering on tissue sections showed a similar picture to the cDNA microarray clusters. All four cluster I genes clustered together, whereas the two cluster II genes clustered on a different branch. Furthermore, all three HCC samples clustered together and the four nontumor liver samples formed a separate cluster. As a whole, our data highlight the power of combining the quantitative, high-throughput cDNA microarray technique to identify novel genes with the traditional pathologic techniques of IHC and ISH to localize expression precisely to individual cell types. The utility of the TreeView software program in the analysis of IHC and ISH data is also underscored.

\section{Discussion}

In this study, we report the analysis of the expression patterns of two endothelial cell cluster genes in HCC using cDNA microarray, RT-PCR, IHC and ISH. We found new sinusoidal endothelial markers in HCC: PODXL, and RGS5. Both are genes found in cluster I which are highly expressed in HCC compared with nontumor liver samples. Cluster II genes, in comparison, has a variable expression pattern in liver tissues.

Interestingly, in the global analysis of the gene expression in HCC, the expression patterns of cluster I endothelial genes correlated positively with the 'proliferation cluster' and 'stromal cells cluster 2', and correlated negatively with 'liverspecific gene cluster' and 'stress response cluster'. The 'proliferation cluster' comprises genes whose functions are required for cell cycle progression, including CDC2, CDC20, and PCNA. The 'liver specific gene cluster' is comprised of genes that are highly expressed in differentiated hepatocytes, for example, cytochrome P450, alcohol dehydrogenase and albumin, etc. The high expression of 'proliferation cluster' genes and the low expression of 'liver specific cluster' genes may represent a hyperproliferative and dedifferentiated state in HCC compared with nontumor liver tissues. And the associated upregulation in cluster I genes may reflect the corresponding alterations in the sinusoidal endothelial cells during malignant transformation. For example, the sinusoidal endothelium of the normal liver is designed to facilitate the exchange of 


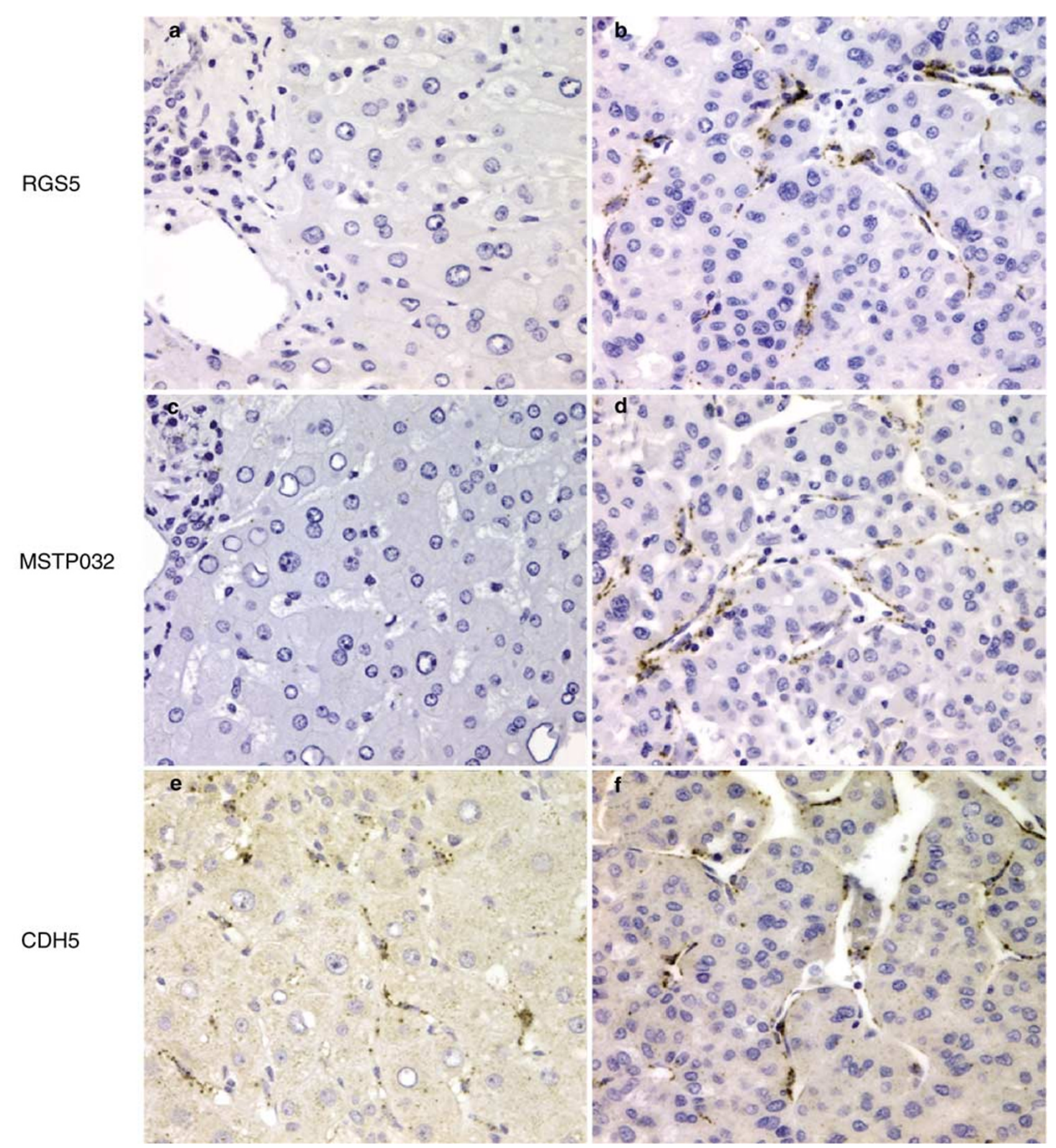

Figure 6 ISH analysis of endothelial cell markers in liver tissues. Riboprobe was used and brown grains denote positive signals. (a), (c), and (e) are normal liver sections; whereas (b), (d), and (f) are HCC sections. (a) and (b) were stained with antisense probe of RGS5 (cluster I); (c) and (d) with antisense probe for MSTP032 (cluster I); (e) and (f) with antisense probe CDH5 (cluster II). All sense control probes for each gene showed no signal (data not shown). The cluster I genes, RGS5 and MSTP032, show strong expression in the endothelium of the HCC but not in the normal liver, whereas the cluster II gene, CDH5, shows strong expression in both the HCC and in the normal liver sinusoids.

compounds with the blood such that liver-specific functions can be performed, while, in contrast, the sinusoidal endothelium in HCC may have a more primitive function to perfuse the growing tumor. The changes in the sinusoidal endothelium in HCC may also help to account for the characteristic 'tumor blush' seen on hepatic angiograms.
The positive correlation of cluster I genes with stromal cell cluster 2, which included COL1A2 and COL3A1, may be related to expression of cluster I genes by stromal cells. In fact, some of the cluster I genes, including SPARC, THY1 and COL4A2, were found to be expressed in human fibroblast as well as endothelial cells using cDNA microarrays. ${ }^{43}$ With in 
1208

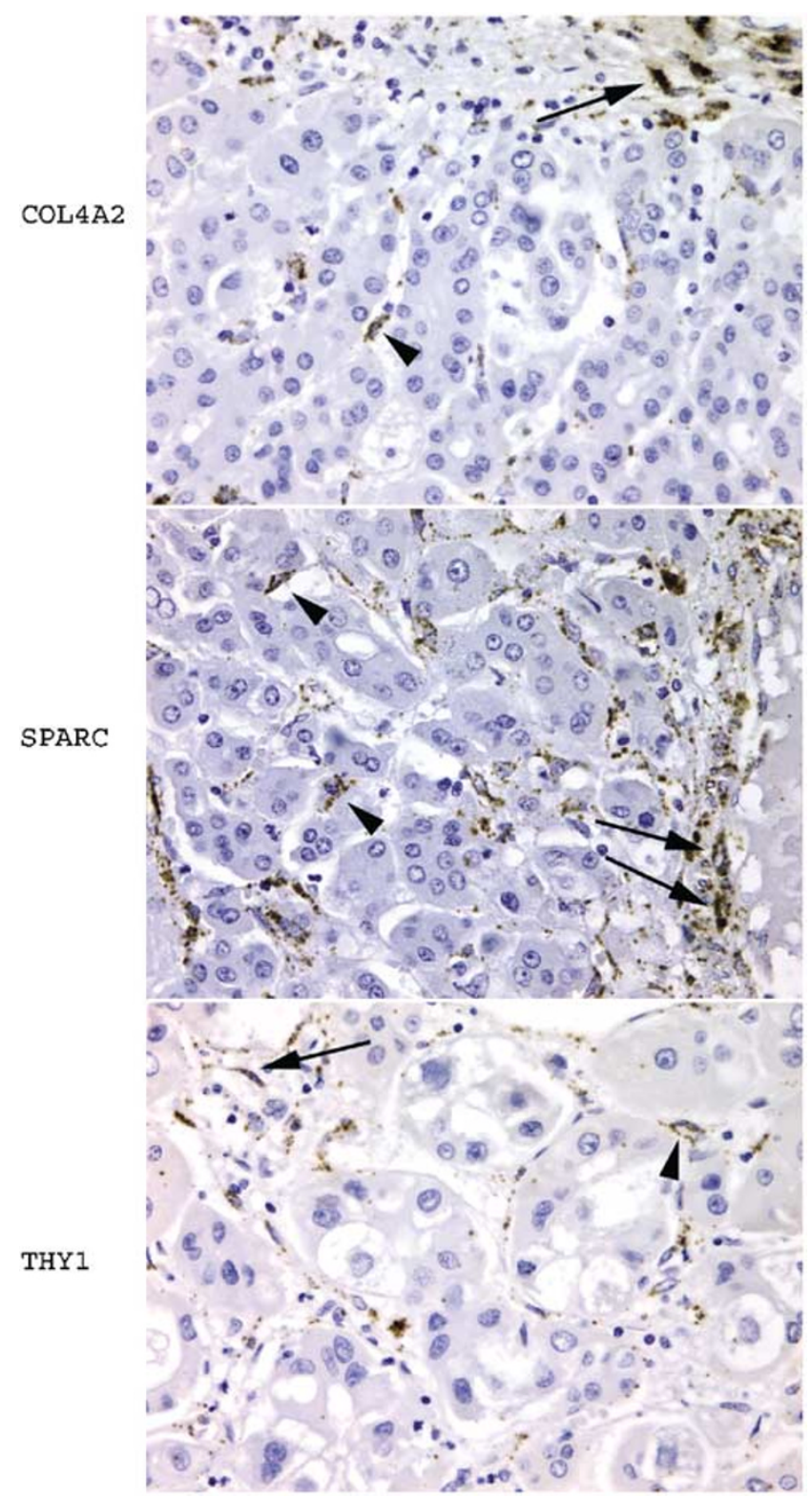

Figure 7 ISH shows that COL4A2, SPARC, and THY1 are expressed in both endothelial cells and stromal cells in HCC. Riboprobe was used and brown grains denoted positive signals. From the top are antisense probe staining of COL4A2, SPARC, and THY1, respectively. Arrowheads indicate endothelial cell staining, whereas arrows indicate stromal cell staining.

situ hybridization, we indeed found the expression of these three genes in both the endothelial cells and stromal cells in HCC (Figure 7). Genes in the 'stress response cluster', which was negatively correlated with cluster I genes, included FOS, JunB, and EGR1. It is possible that alteration of gene expression patterns of the sinusoidal endothelial cells in HCC was associated with increased blood flow to tumor tissues, making the HCC samples less sensitive to environmental stress compared with surrounding normal tissues. This may result in a negative correlation between the expression of cluster I genes and 'stress response cluster' genes.
In our study, we found that the normal sinusoidal endothelium in the liver is less likely to express the conventional vascular markers that are strongly expressed by the endothelium that lines the larger arteries and veins. This finding suggests that the functions performed by these different endothelial cells differ and hence the different gene expression profiles of the endothelium in each different setting. Our current understanding of endothelial cell diversity is still very limited. A recent study revealed the heterogeneity among endothelial cells by using cDNA microarrays to profile gene expression patterns of endothelial cells from different parts of the body. The study identified several groups of genes with tissue-restricted expression among endothelial cells, and suggested that different tissues have specialized endothelial cells with distinct gene expression profiles that may be important for their physiological functions and development. ${ }^{44}$ Sinusoidal endothelial cells in liver are highly specialized, containing many small fenestrations to allow the free diffusion of substances between the hepatocytes and the peripheral blood. ${ }^{10}$ It would be interesting and important to isolate these sinusoidal endothelial cells ${ }^{45}$ and compare the gene expression patterns of these hepatic sinusoidal endothelial cells with endothelial cells from different parts of human body. This would certainly help to illustrate the molecular function, as well as the molecular differentiation program of these specialized endothelial cells in both normal livers and HCC.

This study focused on the expression of the endothelial cell markers of primary HCC compared with normal and cirrhotic livers. It would be interesting to expand the study to examine other primary malignant and benign liver tumors (such as fibrolamellar HCC, hepatoblastoma, cholangiocarcinoma, adenoma, focal nodular hyperplasia, hemangioma), secondary metastatic tumors to the liver, as well as HCC that has spread to distant sites. We believe that the approach of studying gene expression profiles using cDNA microarrays, coupled with validation using RT-PCR, IHC, and ISH will provide a comprehensive analysis and comparison of the endothelial cell markers between these tumors.

In the recent years, significant progress has been made in the field of tumor angiogenesis. However, most studies have focused on endothelial growth factors and their downstream signaling pathways. ${ }^{16,46}$ Little is known about the molecular signature of the endothelial cells that line the blood vessels in tumors. St Croix et $a{ }^{35}$ reported the isolation of endothelial cells from human colorectal tumors and identified several tumor endothelial markers (TEMs) in these cells using SAGE. Further analysis of TEMs in mouse tumors, embryos, and adult tissues revealed that many of them were expressed at high levels in tumor vessels and in the vasculature of the developing embryos, but not in endothelial cells from adult tissues. ${ }^{47}$ These studies clearly demonstrated that endothelial cells in tumor 
tissues have distinct molecular markers. Here, we report the identification of novel endothelial cell markers that are highly expressed in HCC, including PODXL, RGS5, and MSTP032. Our study has further expanded the tumor endothelial cell marker pool. Further investigation of these tumor endothelial cell markers will help achieve a better understanding of the mechanisms involved in tumor angiogenesis.

There have been reports that quantitation of intratumor microvessel density (MVD) using endothelial cell markers may be used as a prognostic marker in various types of tumors, including breast, colon, and pancreatic cancer. ${ }^{48-50}$ In HCC, several groups have reported that high tumor MVD by CD34 or vWF immunostaining may serve as an adverse prognostic marker for HCC less than $5 \mathrm{~cm} .^{51,52}$ Some suggested that higher tumor MVD may predispose to micrometastasis and tumor angiogenesis may be one of the most important factors to influence tumor recurrence of small HCC. Therefore, it would be interesting to assess whether the MVD measured with the novel markers reported in the current study may prove to be a better and more reliable prognostic marker than CD34. Alternatively, one or more of the novel sinusoidal endothelial markers may enhance the predictive value when used in combination with CD34.

The identification of endothelial cell markers that are highly expressed in HCC may have other potential applications in the diagnosis and treatment of HCC. One example is in the field of molecular imaging. Early detection and early surgical resection is highly correlated with survival rate in HCC patients. Unfortunately, current screening methods using serum alpha-fetoprotein and imaging technologies such as CAT scan and MRI still miss a significant number of small HCC. ${ }^{53}$ There is a great need for more sensitive and specific imaging technology for the accurate and early diagnosis of HCC. Radioimmunoscintigraphy (RIS) is a relatively new and rapidly developing new technology that takes advantage of the antibody specificity of tumor surface antigens and of the emitted radiation from suitable radioisotopes as the mean of imaging. ${ }^{54}$ One of the most crucial steps towards the success of RIS is the choice of antibodies that can recognize tumorspecific antigens. Antibodies against these protein products of the novel endothelial cell markers may therefore be useful in RIS and provide a more specific and sensitive method for the detection and treatment of HCC.

\section{Acknowledgements}

We are grateful to the members of the Patrick Brown Lab in the Department of Biochemistry, Stanford University. We thank Dr Christi Iacobuzio-Donahue from Johns Hopkins University for the help with ISH, and Doug Ross from Applied Genomics who provided us with the antibody to PODXL. We also thank the Stanford Functional Genomic Center,
Stanford Microarray database, and Stanford Asian Liver Center for their support. The study is supported in part by a grant to the Asian Liver Center at Stanford University by the HM Liu Foundation (to XC, RL, VM, and SS) and UCSF Liver Center (DK26743-22) pilot/feasibility project award (to XC).

\section{References}

1 Pisani P, Parkin DM, Bray F, et al. Estimates of the worldwide mortality from 25 cancers in 1990. Int J Cancer 1999;83:18-29.

2 Simonetti RG, Camma C, Fiorello F, et al. Hepatocellular carcinoma. A worldwide problem and the major risk factors. Dig Dis Sci 1991;36:962-972.

3 Feitelson MA, Sun B, Satiroglu Tufan NL, et al. Genetic mechanisms of hepatocarcinogenesis. Oncogene 2002;21:2593-2604.

4 Tsukuma H, Hiyama T, Tanaka S, et al. Risk factors for hepatocellular carcinoma among patients with chronic liver disease. N Engl J Med 1993;328:1797-1801.

5 El-Serag HB, Mason AC. Rising incidence of hepatocellular carcinoma in the United States. N Engl J Med 1999;340:745-750.

6 Lin TY, Lee CS, Chen KM, et al. Role of surgery in the treatment of primary carcinoma of the liver: a 31-year experience. Br J Surg 1987;74:839-842.

7 Mor E, Kaspa RT, Sheiner P, et al. Treatment of hepatocellular carcinoma associated with cirrhosis in the era of liver transplantation. Ann Intern Med 1998;129:643-653.

8 El-Serag HB, Mason AC, Key C. Trends in survival of patients with hepatocellular carcinoma between 1977 and 1996 in the United States. Hepatology 2001;33: 62-65.

9 Bouwens L, De Bleser P, Vanderkerken K, et al. Liver cell heterogeneity: functions of non-parenchymal cells. Enzyme 1992;46:155-168.

10 Arii S, Imamura M. Physiological role of sinusoidal endothelial cells and Kupffer cells and their implication in the pathogenesis of liver injury. J Hepatobiliary Pancreat Surg 2000;7:40-48.

11 Folkman J. Fundamental concepts of the angiogenic process. Curr Mol Med 2003;3:643-651.

12 Bergers G, Benjamin LE. Tumorigenesis and the angiogenic switch. Nat Rev Cancer 2003;3:401-410.

13 Sugimachi K, Tanaka S, Terashi T, et al. The mechanisms of angiogenesis in hepatocellular carcinoma: angiogenic switch during tumor progression. Surgery 2002;131:S135-S141.

14 Mitsuhashi N, Shimizu H, Ohtsuka M, et al. Angiopoietins and Tie-2 expression in angiogenesis and proliferation of human hepatocellular carcinoma. Hepatology 2003;37:1105-1113.

15 Hashizume H, Baluk P, Morikawa S, et al. Openings between defective endothelial cells explain tumor vessel leakiness. Am J Pathol 2000;156:1363-1380.

16 Dvorak HF. Vascular permeability factor/vascular endothelial growth factor: a critical cytokine in tumor angiogenesis and a potential target for diagnosis and therapy. J Clin Oncol 2002;20:4368-4380.

17 Folkman J. Role of angiogenesis in tumor growth and metastasis. Semin Oncol 2002;29:15-18.

18 Ruck P, Xiao JC, Kaiserling E. Immunoreactivity of sinusoids in hepatocellular carcinoma. An immuno- 
histochemical study using lectin UEA-1 and antibodies against endothelial markers, including CD34. Arch Pathol Lab Med 1995;119:173-178.

19 Cui S, Hano H, Sakata A, et al. Enhanced CD34 expression of sinusoid-like vascular endothelial cells in hepatocellular carcinoma. Pathol Int 1996;46:751-756.

20 Welsh JB, Zarrinkar PP, Sapinoso LM, et al. Analysis of gene expression profiles in normal and neoplastic ovarian tissue samples identifies candidate molecular markers of epithelial ovarian cancer. Proc Natl Acad Sci USA 2001;98:1176-1181.

21 Leung SY, Chen X, Chu KM, et al. Phospholipase A2 group IIA expression in gastric adenocarcinoma is associated with prolonged survival and less frequent metastasis. Proc Natl Acad Sci USA 2002;99: 16203-16208.

22 Dhanasekaran SM, Barrette TR, Ghosh D, et al. Delineation of prognostic biomarkers in prostate cancer. Nature 2001;412:822-826.

23 van't Veer LJ, Dai H, van de Vijver MJ, et al. Gene expression profiling predicts clinical outcome of breast cancer. Nature 2002;415:530-536.

24 Chen X, Cheung ST, So S, et al. Gene expression patterns in human liver cancers. Mol Biol Cell 2002;13:1929-1939.

25 Cheung ST, Chen X, Guan XY, et al. Identify metastasis-associated genes in hepatocellular carcinoma through clonality delineation for multinodular tumor. Cancer Res 2002;62:4711-4721.

26 Sherlock G, Hernandez-Boussard T, Kasarskis A, et al. The Stanford microarray database. Nucleic Acids Res 2001;29:152-155.

27 Eisen MB, Spellman PT, Brown PO, et al. Cluster analysis and display of genome-wide expression patterns. Proc Natl Acad Sci USA 1998;95:14863-14868.

28 Whitney AR, Diehn M, Popper SJ, et al. Individuality and variation in gene expression patterns in human blood. Proc Natl Acad Sci USA 2003;100:1896-1901.

29 Higgins JP, Warnke RA. CD30 expression is common in mediastinal large B-cell lymphoma. Am J Clin Pathol 1999;112:241-247.

30 Kononen J, Bubendorf L, Kallioniemi A, et al. Tissue microarrays for high-throughput molecular profiling of tumor specimens. Nat Med 1998;4:844-847.

31 Van De Rijn M, Perou CM, Tibshirani R, et al. Expression of cytokeratins 17 and 5 identifies a group of breast carcinomas with poor clinical outcome. Am J Pathol 2002;161:1991-1996.

32 Higgins JP, Montgomery K, Wang L, et al. Expression of FKBP12 in benign and malignant vascular endothelium: an immunohistochemical study on conventional sections and tissue microarrays. Am J Surg Pathol 2003;27:58-64.

33 Liu CL, Prapong W, Natkunam Y, et al. Software tools for high-throughput analysis and archiving of immunohistochemistry staining data obtained with tissue microarrays. Am J Pathol 2002;161:1557-1565.

34 Iacobuzio-Donahue CA, Argani P, Hempen PM, et al. The desmoplastic response to infiltrating breast carcinoma: gene expression at the site of primary invasion and implications for comparisons between tumor types. Cancer Res 2002;62:5351-5357.

35 St Croix B, Rago C, Velculescu V, et al. Genes expressed in human tumor endothelium. Science 2000;289:1197-1202.

36 Myers JC, Dion AS, Abraham V, et al. Type XV collagen exhibits a widespread distribution in human tissues but a distinct localization in basement membrane zones. Cell Tissue Res 1996;286:493-505.

37 Hagg PM, Hagg PO, Peltonen S, et al. Location of type XV collagen in human tissues and its accumulation in the interstitial matrix of the fibrotic kidney. Am J Pathol 1997;150:2075-2086.

38 Hla T, Maciag T. An abundant transcript induced in differentiating human endothelial cells encodes a polypeptide with structural similarities to G-proteincoupled receptors. J Biol Chem 1990;265:9308-9313.

39 Liu Y, Wada R, Yamashita T, et al. Edg-1, the G proteincoupled receptor for sphingosine-1-phosphate, is essential for vascular maturation. J Clin Invest 2000;106:951-961.

40 Kershaw DB, Thomas PE, Wharram BL, et al. Molecular cloning, expression, and characterization of podocalyxin-like protein 1 from rabbit as a transmembrane protein of glomerular podocytes and vascular endothelium. J Biol Chem 1995;270:29439-29446.

41 Kershaw DB, Beck SG, Wharram BL, et al. Molecular cloning and characterization of human podocalyxinlike protein. Orthologous relationship to rabbit PCLP1 and rat podocalyxin. J Biol Chem 1997;272: 15708-15714.

42 Seki N, Sugano S, Suzuki Y, et al. Isolation, tissue expression, and chromosomal assignment of human RGS5, a novel G-protein signaling regulator gene. J Hum Genet 1998;43:202-205.

43 Ji J, Chen X, Leung SY, et al. Comprehensive analysis of the gene expression profiles in human gastric cancer cell lines. Oncogene 2002;21:6549-6556.

44 Chi JT, Chang HY, Haraldsen G, et al. Endothelial cell diversity revealed by global expression profiling. Proc Natl Acad Sci USA 2003;100:10623-10628.

45 David H, Kassner G, Krause W, et al. Ultrastructure and quantitative composition of isolated endothelial cells of rat liver. Exp Pathol 1990;39:95-101.

46 Carmeliet P, Collen D. Molecular basis of angiogenesis. Role of VEGF and VE-cadherin. Ann NY Acad Sci 2000;902:249-262 (discussion 262-4).

47 Carson-Walter EB, Watkins DN, Nanda A, et al. Cell surface tumor endothelial markers are conserved in mice and humans. Cancer Res 2001;61:6649-6655.

48 Acenero MJ, Gonzalez JF, Gallego MG, et al. Vascular enumeration as a significant prognosticator for invasive breast carcinoma. J Clin Oncol 1998;16:1684-1688.

49 Frank RE, Saclarides TJ, Leurgans S, et al. Tumor angiogenesis as a predictor of recurrence and survival in patients with node-negative colon cancer. Ann Surg 1995;222:695-699.

50 Niedergethmann M, Hildenbrand R, Wolf G, et al. Angiogenesis and cathepsin expression are prognostic factors in pancreatic adenocarcinoma after curative resection. Int J Pancreatol 2000;28:31-39.

51 Poon RT, Ng IO, Lau C, et al. Tumor microvessel density as a predictor of recurrence after resection of hepatocellular carcinoma: a prospective study. J Clin Oncol 2002;20:1775-1785.

52 Sun HC, Tang ZY, Li XM, et al. Microvessel density of hepatocellular carcinoma: its relationship with prognosis. J Cancer Res Clin Oncol 1999;125:419-426.

53 Nguyen MH, Keeffe EB. Screening for hepatocellular carcinoma. J Clin Gastroenterol 2002;35:S86-S91.

54 Potamianos S, Varvarigou AD, Archimandritis SC. Radioimmunoscintigraphy and radioimmunotherapy in cancer: principles and application. Anticancer Res 2000;20:925-948. 